\title{
ADI Finite Difference Discretization of the Heston-Hull-White PDE
}

\author{
Tinne Haentjens and Karel in 't Hout \\ Department of Mathematics and Computer Science, University of Antwerp, \\ Middelheimlaan 1, 2020 Antwerp, Belgium
}

\begin{abstract}
This paper concerns the efficient numerical solution of the time-dependent, three-dimensional Heston-Hull-White PDE for the fair prices of European call options. The numerical solution method described in this paper consists of a finite difference discretization on non-uniform spatial grids followed by an Alternating Direction Implicit scheme for the time discretization and extends the method recently proved effective by In 't Hout \& Foulon (2010) for the simpler, two-dimensional Heston PDE.
\end{abstract}

Keywords: financial option pricing, Heston model, Hull-White model, finite difference methods, ADI schemes.

PACS: $02.60 . \mathrm{Lj}, 02.70 . \mathrm{Bf}, 89.65 . \mathrm{Gh}$

\section{INTRODUCTION: OPTION PRICE MODEL}

We consider the numerical valuation of European call options in a general asset price model given by the system of stochastic differential equations (SDEs)

$$
\left\{\begin{array}{l}
d S_{\tau}=R_{\tau} S_{\tau} d \tau+\sqrt{V_{\tau}} S_{\tau} d W_{\tau}^{1} \\
d V_{\tau}=\kappa\left(\eta-V_{\tau}\right) d \tau+\sigma_{1} \sqrt{V_{\tau}} d W_{\tau}^{2}, \\
d R_{\tau}=a\left(b(\tau)-R_{\tau}\right) d \tau+\sigma_{2} d W_{\tau}^{3}
\end{array}\right.
$$

for $0<\tau \leq T$ with $T>0$ the given maturity time of the option. Here $S_{\tau}, V_{\tau}, R_{\tau}$ denote random variables that represent the asset price, its variance, and the interest rate, respectively, at time $\tau$. The asset price model (1) constitutes a natural extension of the well-known Black-Scholes model where the volatility $\sqrt{V_{\tau}}$ and the interest rate both evolve randomly over time. The stochastic models for $V_{\tau}$ and $R_{\tau}$ were proposed by Heston and Hull \& White, respectively, cf. [5, 6, 7]. The quantities $\kappa, \eta, \sigma_{1}$ and $a, \sigma_{2}$ are given positive real constants; $b$ is a given deterministic, positive function of time; $W_{\tau}^{1}, W_{\tau}^{2}, W_{\tau}^{3}$ are Brownian motions, with (arbitrary) given correlation factors $\rho_{12}, \rho_{13}, \rho_{23} \in[-1,1]$.

Let $u(s, v, r, t)$ denote the fair price of a European call option if at time $\tau=T-t$ the asset price equals $s$, its variance equals $v$ and the interest rate equals $r$. If $K>0$ is the given strike price of the option, then the payoff defines

$$
u(s, v, r, 0)=\max (0, s-K) .
$$

Financial option pricing theory yields that, under the model (1), the price for $0<t \leq T$ is given by the expected value

$$
u(s, v, r, t)=\mathbb{E}\left[e^{-\int_{T-t}^{T} R_{\tau} d \tau} \max \left(0, S_{T}-K\right) \mid S_{T-t}=s, V_{T-t}=v, R_{T-t}=r\right]
$$

and satisfies the following partial differential equation (PDE),

$$
\begin{aligned}
\frac{\partial u}{\partial t}= & \frac{1}{2} s^{2} v \frac{\partial^{2} u}{\partial s^{2}}+\frac{1}{2} \sigma_{1}^{2} v \frac{\partial^{2} u}{\partial v^{2}}+\frac{1}{2} \sigma_{2}^{2} \frac{\partial^{2} u}{\partial r^{2}}+\rho_{12} \sigma_{1} s v \frac{\partial^{2} u}{\partial s \partial v}+\rho_{13} \sigma_{2} s \sqrt{v} \frac{\partial^{2} u}{\partial s \partial r} \\
& +\rho_{23} \sigma_{1} \sigma_{2} \sqrt{v} \frac{\partial^{2} u}{\partial v \partial r}+r s \frac{\partial u}{\partial s}+\kappa(\eta-v) \frac{\partial u}{\partial v}+a(b(T-t)-r) \frac{\partial u}{\partial r}-r u
\end{aligned}
$$

for $s>0, v>0,-\infty<r<\infty$. We refer to (3) as the Heston-Hull-White (HHW) PDE. It can be viewed as a timedependent convection-diffusion-reaction equation on an unbounded, three-dimensional spatial domain.

The aim of this paper is to describe an efficient numerical solution method for the HHW PDE. We follow the methodof-lines approach. For the space discretization, we use finite difference (FD) schemes on non-uniform Cartesian grids 
in the $(s, v, r)$-domain. For the time discretization, we apply a specific Alternating Direction Implicit (ADI) scheme that is tailored to semi-discrete systems stemming from multi-dimensional PDEs with mixed spatial-derivative terms. The numerical solution method formulated in this paper for the HHW PDE extends the method that has recently been proved effective [2] for the simpler, two-dimensional Heston PDE.

\section{SPACE DISCRETIZATION OF THE HESTON-HULL-WHITE PDE}

As a preliminary step, the spatial domain is restricted to a bounded set $\left[0, S_{\max }\right] \times\left[0, V_{\max }\right] \times\left[-R_{\max }, R_{\max }\right]$ with fixed values $S_{\max }, V_{\max }, R_{\max }$ chosen sufficiently large. The following boundary conditions are imposed,

$$
\begin{aligned}
u(0, v, r, t) & =0, & & \frac{\partial u}{\partial r}\left(s, v,-R_{\max }, t\right)=0, \\
\frac{\partial u}{\partial s}\left(S_{\max }, v, r, t\right) & =1, & u\left(s, V_{\max }, r, t\right)=s, & \frac{\partial u}{\partial r}\left(s, v, R_{\max }, t\right)=0 .
\end{aligned}
$$

Clearly, these conditions are of Dirichlet and Neumann type. We assume throughout that the Feller condition $2 \kappa \eta>\sigma_{1}^{2}$ holds, which implies that $v=0$ forms an outflow boundary and no information concerning $u$ is required there.

For the space discretization of the initial-boundary value problem (2)-(4) for the HHW PDE we consider an extension of the FD discretization on Cartesian grids described in [2] for the two-dimensional Heston PDE. The spatial grid is such that in the $s$ - and $v$-directions non-uniform meshes $0=s_{0}<s_{1}<\ldots<s_{m_{1}}=S_{\max }$ and $0=v_{0}<$ $v_{1}<\ldots<v_{m_{2}}=V_{\max }$ are defined with relatively many points in the neighborhood of $s=K$ and $v=0$, respectively. This choice is natural for numerical reasons, since the initial function (2) is non-smooth at $s=K$ and for $v \approx 0$ the HHW PDE is convection-dominated in the $v$-direction. Moreover, values $(s, v)$ that lie near $(K, 0)$ are of main practical interest. In the $r$-direction, we define a non-uniform mesh as well, where many points are placed near the so-called reversion level $b(t)$ at each time $t$. For ease of presentation, we suppose here that $b$ is constant, $b(t)=b$. Let integer $m_{3} \geq 1$ and parameter $c>0$ and let equidistant $\xi_{0}<\xi_{1}<\ldots<\xi_{m_{3}}$ be given by

$\xi_{k}=\sinh ^{-1}\left(\left(-R_{\max }-b\right) / c\right)+k \cdot \Delta \xi \quad\left(0 \leq k \leq m_{3}\right) \quad$ with $\Delta \xi=\frac{1}{m_{3}}\left[\sinh ^{-1}\left(\left(R_{\max }-b\right) / c\right)-\sinh ^{-1}\left(\left(-R_{\max }-b\right) / c\right)\right]$.

Then a non-uniform mesh $-R_{\max }=r_{0}<r_{1}<\ldots<r_{m_{3}}=R_{\max }$ is defined through the transformation

$$
r_{k}=b+c \sinh \left(\xi_{k}\right) \quad\left(0 \leq k \leq m_{3}\right)
$$

The parameter $c$ controls the relative number of mesh points $r_{k}$ that lie near the reversion level $b$. The above mesh is smooth in the sense that there exist real constants $C_{0}, C_{1}, C_{2}>0$ such that the mesh widths $\Delta r_{k}=r_{k}-r_{k-1}$ satisfy

$$
\left.C_{0} \Delta \xi \leq \Delta r_{k} \leq C_{1} \Delta \xi \quad \text { and } \quad\left|\Delta r_{k+1}-\Delta r_{k}\right| \leq C_{2}(\Delta \xi)^{2} \quad \text { (uniformly in } k, m_{3}\right) \text {. }
$$

The type of mesh for $r$ is the same as employed in [2] for $s$ and $v$. It has previously been considered e.g. in [7]. In our application, we have chosen $c=K / 10, c=V_{\max } / 500, c=R_{\max } / 400$ for the $s$-, $v$-, $r$-directions, respectively.

To formulate the FD schemes under consideration, let $f: \mathbb{R} \rightarrow \mathbb{R}$ be any given function, let $\left\{x_{i}\right\}_{i \in \mathbb{Z}}$ be any given increasing sequence of mesh points, and $\Delta x_{i}=x_{i}-x_{i-1}$ for all $i$. We use the following FD approximations of the derivatives $f^{\prime}\left(x_{i}\right)$ and $f^{\prime \prime}\left(x_{i}\right)$ :

$$
\begin{aligned}
& f^{\prime}\left(x_{i}\right) \approx \frac{\Delta x_{i}}{\Delta x_{i-1}\left(\Delta x_{i-1}+\Delta x_{i}\right)} f\left(x_{i-2}\right)+\frac{-\Delta x_{i-1}-\Delta x_{i}}{\Delta x_{i-1} \Delta x_{i}} f\left(x_{i-1}\right)+\frac{\Delta x_{i-1}+2 \Delta x_{i}}{\Delta x_{i}\left(\Delta x_{i-1}+\Delta x_{i}\right)} f\left(x_{i}\right), \\
& f^{\prime}\left(x_{i}\right) \approx \frac{-\Delta x_{i+1}}{\Delta x_{i}\left(\Delta x_{i}+\Delta x_{i+1}\right)} f\left(x_{i-1}\right)+\frac{\Delta x_{i+1}-\Delta x_{i}}{\Delta x_{i} \Delta x_{i+1}} f\left(x_{i}\right)+\frac{\Delta x_{i}}{\Delta x_{i+1}\left(\Delta x_{i}+\Delta x_{i+1}\right)} f\left(x_{i+1}\right), \\
& f^{\prime}\left(x_{i}\right) \approx \frac{-2 \Delta x_{i+1}-\Delta x_{i+2}}{\Delta x_{i+1}\left(\Delta x_{i+1}+\Delta x_{i+2}\right)} f\left(x_{i}\right)+\frac{\Delta x_{i+1}+\Delta x_{i+2}}{\Delta x_{i+1} \Delta x_{i+2}} f\left(x_{i+1}\right)+\frac{-\Delta x_{i+1}}{\Delta x_{i+2}\left(\Delta x_{i+1}+\Delta x_{i+2}\right)} f\left(x_{i+2}\right), \\
& f^{\prime \prime}\left(x_{i}\right) \approx \frac{2}{\Delta x_{i}\left(\Delta x_{i}+\Delta x_{i+1}\right)} f\left(x_{i-1}\right)+\frac{-2}{\Delta x_{i} \Delta x_{i+1}} f\left(x_{i}\right) \quad+\frac{2}{\Delta x_{i+1}\left(\Delta x_{i}+\Delta x_{i+1}\right)} f\left(x_{i+1}\right) .
\end{aligned}
$$

Note that $(5 \mathrm{~b}),(5 \mathrm{~d})$ are central schemes and $(5 \mathrm{a}),(5 \mathrm{c})$ are upwind schemes. If $f: \mathbb{R}^{2} \rightarrow \mathbb{R}$ is any given function of two variables $(x, y)$, then we approximate the mixed derivative $f_{x y}\left(x_{i}, y_{j}\right)$ at any point $\left(x_{i}, y_{j}\right)$ by successive application 
of (5b) in the $x$ - and $y$-directions. This is equivalent to a FD approximation based on a 9-point stencil centered about $\left(x_{i}, y_{j}\right)$. For smooth non-uniform meshes, all FD schemes above possess a second-order truncation error.

The actual FD discretization of (2)-(4) is performed as follows. In view of the Dirichlet boundary conditions in (4), the relevant set of grid points is $\mathscr{G}=\left\{\left(s_{i}, v_{j}, r_{k}\right): 1 \leq i \leq m_{1}, 0 \leq j \leq m_{2}-1,0 \leq k \leq m_{3}\right\}$. At this grid, each spatial derivative appearing in (3) is replaced by its corresponding central FD approximation, except:

- At the boundary $s=S_{\max }$ the derivatives in the $s$-direction need to be considered. The Neumann condition clearly yields $\partial u / \partial s$ and it implies that the mixed derivatives $\partial^{2} u / \partial s \partial v, \partial^{2} u / \partial s \partial r$ vanish there. Next, $\partial^{2} u / \partial s^{2}$ is approximated at $s=S_{\max }$ by (5d) using a virtual point $s_{m_{1}+1}>S_{\max }$ where the value at this point is defined by extrapolation.

- At the outflow boundary $v=0$ the derivative $\partial u / \partial v$ is approximated by the upwind scheme $(5 \mathrm{c})$. All other derivatives in the $v$-direction have a factor $v$ or $\sqrt{v}$ in front in (3), and thus can conveniently be set equal to zero.

- In the region $v>1$, we apply the upwind scheme (5a) for $\partial u / \partial v$ whenever the flow in the $v$-direction is towards $v=V_{\max }$. This is done so as to avoid spurious oscillations in the FD solution when $\sigma_{1}$ is small.

- At the boundaries $r= \pm R_{\max }$ the Neumann conditions are incorporated similarly as for $s$ at the boundary $s=S_{\max }$.

The FD discretization of the initial-boundary value problem for the HHW PDE gives rise to an initial value problem for a large system of stiff ordinary differential equations (ODEs),

$$
U^{\prime}(t)=A(t) U(t)+g(t) \quad(0 \leq t \leq T), \quad U(0)=U_{0} .
$$

Here $A(t)$, for any $t \geq 0$, is a given real $m \times m$-matrix and $g(t), U_{0}$ are given real $m$-vectors, with $m=m_{1} m_{2}\left(m_{3}+1\right)$. The vector function $g$ depends on the boundary conditions (4) and the vector $U_{0}$ is directly obtained from the initial condition (2). For each $t>0$, the entries of the solution vector $U(t)$ to (6) form approximations to the exact option values $u(s, v, r, t)$ at the grid points $(s, v, r) \in \mathscr{G}$, ordered in a convenient way.

\section{TIME DISCRETIZATION: THE MODIFIED CRAIG-SNEYD SCHEME}

The selection of a suitable time discretization scheme for the semi-discrete HHW system (6) is crucial to acquiring an effective full numerical solution method for the HHW initial-boundary value problem. Standard implicit schemes such as Crank-Nicolson are in general computationally too demanding. For the efficient time discretization of (6), we propose in this paper a splitting scheme of the ADI type. We decompose the matrix $A(t)$ into four simpler matrices,

$$
A(t)=A_{0}+A_{1}+A_{2}+A_{3}(t) .
$$

Here $A_{0}$ represents the part of $A(t)$ that stems from the FD discretization of all mixed derivative terms in the HHW PDE. Note that $A_{0}$ is nonzero whenever one of the correlation factors $\rho_{12}, \rho_{13}, \rho_{23}$ is nonzero. In line with the traditional ADI idea, the matrices $A_{1}, A_{2}, A_{3}(t)$ represent the parts of $A(t)$ that stem from the FD discretization of all spatial derivatives in the $s-, v$-and $r$-directions, respectively, and the $r u$ term from (3) is distributed evenly over $A_{1}, A_{2}, A_{3}(t)$. We decompose $g(t)=g_{0}+g_{1}+g_{2}+g_{3}(t)$ analogous to that of $A(t)$.

Let $\theta>0$ be a given real parameter, let $\Delta t=T / N$ with integer $N \geq 1$, and $t_{n}=n \cdot \Delta t$. The following scheme generates, in a one-step manner, approximations $U_{n}$ to the exact solution values $U\left(t_{n}\right)$ of (6) for $n=1,2, \ldots, N$ :

$$
\left\{\begin{array}{l}
Y_{0}=U_{n-1}+\Delta t\left(A\left(t_{n-1}\right) U_{n-1}+g\left(t_{n-1}\right)\right) \\
Y_{j}=Y_{j-1}+\theta \Delta t A_{j}\left(Y_{j}-U_{n-1}\right) \quad(j=1,2) \\
Y_{3}=Y_{2}+\theta \Delta t\left(A_{3}\left(t_{n}\right) Y_{3}-A_{3}\left(t_{n-1}\right) U_{n-1}+g_{3}\left(t_{n}\right)-g_{3}\left(t_{n-1}\right)\right) \\
\widehat{Y}_{0}=Y_{0}+\theta \Delta t A_{0}\left(Y_{3}-U_{n-1}\right) \\
\widetilde{Y}_{0}=\widehat{Y}_{0}+\left(\frac{1}{2}-\theta\right) \Delta t\left(A\left(t_{n}\right) Y_{3}-A\left(t_{n-1}\right) U_{n-1}+g\left(t_{n}\right)-g\left(t_{n-1}\right)\right) \\
\widetilde{Y}_{j}=\widetilde{Y}_{j-1}+\theta \Delta t A_{j}\left(\widetilde{Y}_{j}-U_{n-1}\right) \quad(j=1,2) \\
\widetilde{Y}_{3}=\widetilde{Y}_{2}+\theta \Delta t\left(A_{3}\left(t_{n}\right) \widetilde{Y}_{3}-A_{3}\left(t_{n-1}\right) U_{n-1}+g_{3}\left(t_{n}\right)-g_{3}\left(t_{n-1}\right)\right) \\
U_{n}=\widetilde{Y}_{3}
\end{array}\right.
$$



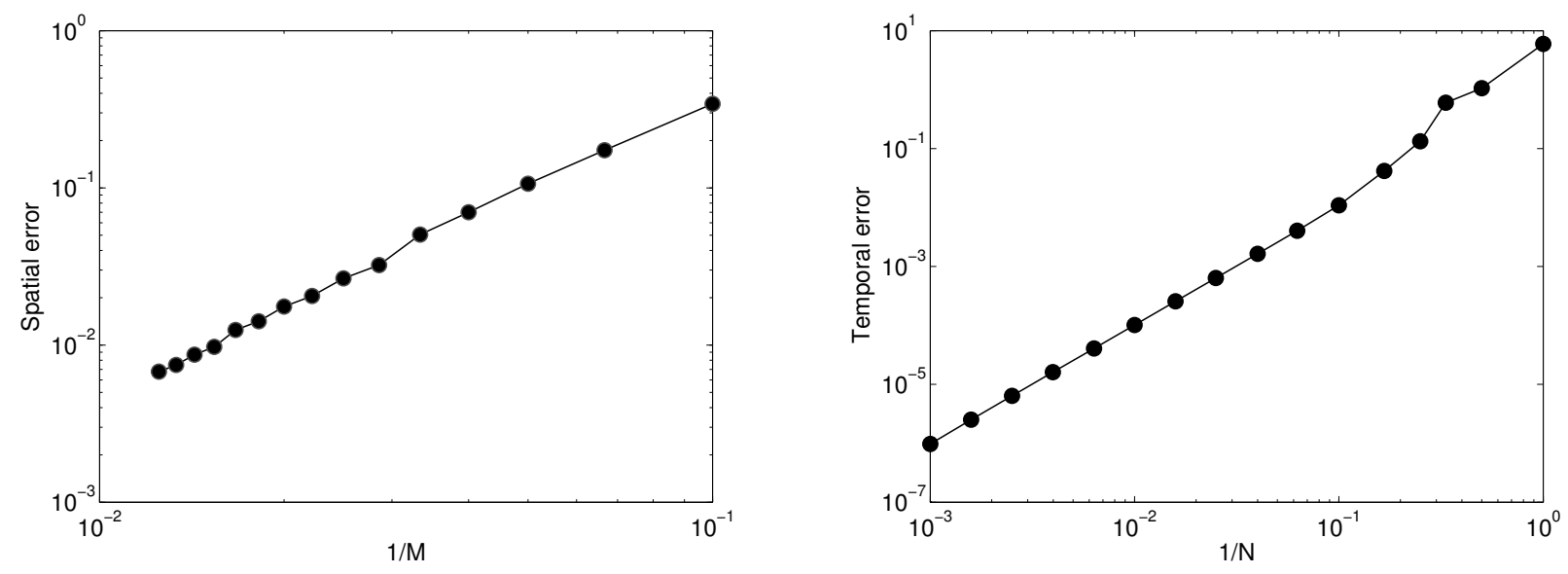

The scheme (8) is called the Modified Craig-Sneyd (MCS) scheme. It has recently been introduced, in a slightly more general form, by In 't Hout \& Welfert [4]. Taylor expansion yields that the MCS scheme has a classical order of consistency equal to two for any given $\theta$. A perusal of (8) shows that the $A_{0}$ part, representing all mixed derivative terms, is always treated in an explicit fashion. The $A_{1}, A_{2}, A_{3}(t)$ parts are successively treated in an implicit fashion.

The MCS scheme retains the key advantage of traditional ADI schemes in the presence of mixed derivative terms: the arising linear systems are efficiently solved by $L U$ factorization, as the pertinent matrices are essentially tridiagonal or pentadiagonal. For standard implicit methods, the matrices that arise have a (very) large bandwidth and solving by $L U$ factorization is in general too demanding. Moreover, with the splitting (7), the time-dependency of $A$ is only passed onto the simpler matrix $A_{3}$, which yields an additional advantage of (8) over standard implicit methods.

The MCS scheme with $\theta=\frac{1}{3}$ has recently been found effective [2] in the numerical solution of the Heston PDE. In $[3,4]$ positive stability results were proved for the MCS scheme when applied to two-dimensional convection-diffusion equations with mixed derivative terms. A stability analysis that is directly relevant to its application in the case of the three-dimensional HHW PDE is non-trivial and yet incomplete. Ample numerical evidence indicates, however, the interesting result that the MCS scheme with the choice $\theta=\frac{1}{3}$ is also stable and effective in this case. It is our aim of future research to rigorously investigate this. In the following section we provide a numerical illustration.

\section{NUMERICAL EXPERIMENT}

If the Brownian motion $W_{\tau}^{3}$ is not correlated to $W_{\tau}^{1}$ and $W_{\tau}^{2}$ (i.e., if $\rho_{13}=\rho_{23}=0$ ), then a semi closed-form analytic formula for the exact European call option price function $u$ is known [1]. We employ this formula to gain insight into the global spatial error, defined as the maximal absolute error between $u\left(s_{i}, v_{j}, r_{k}, T\right)$ and the corresponding entry of the solution vector $U(T)$ to (6) over a region of interest, chosen here as $\frac{1}{2} K<s_{i}<\frac{3}{2} K, 0<v_{j}<1,0<r_{k}<\frac{1}{4}$. Together with this we study the global temporal error, defined as the maximal absolute error between corresponding entries of $U(T)$ and its approximation $U_{N}$ (where $T=N \cdot \Delta t$ ) over the same region of interest. For the numerical illustration we consider the Heston and Hull-White parameter values

$$
\kappa=3, \quad \eta=0.12, \quad \sigma_{1}=0.04, \quad a=0.1, \quad b(t) \equiv 0.06, \quad \sigma_{2}=0.01, \quad \rho_{12}=0.6, \quad \rho_{13}=0, \quad \rho_{23}=0 .
$$

The maturity time, strike price and spatial domain are taken as $T=1, K=100$ and $[0,14 K] \times[0,5] \times[-4,4]$.

The left part of the figure displays the estimated global spatial errors versus $1 / M$ for $M=10,15, \ldots, 80$ where $m_{1}=2 M, m_{2}=M$ and $m_{3}=M$. The observed order of convergence for the spatial error is approximately equal to two, which is as desired.

The right part of the figure concerns the MCS scheme with $\theta=\frac{1}{3}$. It displays the estimated global temporal errors versus $1 / N$ for a range of step numbers $1 \leq N \leq 1000$ when $M=50$. We note that this scheme was applied with $N=5000$ to obtain a reference solution for $U(T)$. Clearly, the temporal errors are uniformly bounded from above by a moderate value and decay monotonically as $N$ increases. Further experiments show that the temporal errors are only weakly affected by the chosen number of spatial grid points. These observations are compatible with an 
unconditionally stable behavior of the pertinent MCS scheme. Subsequently, a convergence behavior is observed with an order approximately equal to two, which agrees with its classical order of consistency.

We note that the implementation has been done in Matlab, version 7.8, where all matrices were defined as sparse. As an indication for the computing times, our implementation of the MCS scheme took about $0.20 \mathrm{cpu}-\mathrm{sec}$ per time step if $M=50$ (i.e., $m \approx 2.5 \times 10^{5}$ ) on a Intel Core Duo T7250 $2.00 \mathrm{GHz}$ processor with $4 \mathrm{~GB}$ memory.

In addition to the stability analysis mentioned at the end of the previous section, we shall elaborate in future research more on the performance of the numerical solution method described in this paper. Among others, time-dependent reversion levels, correlation factors that are all nonzero, and exotic options will be considered as well as the combined spatial-temporal errors.

\section{ACKNOWLEDGEMENTS}

The authors are grateful to Jan Van Casteren for his valuable remarks on the existence and uniqueness of solutions to the SDE (1) and the validity of the PDE (3). This work has been supported financially by the Research Foundation Flanders, FWO contract no. G.0125.08.

\section{REFERENCES}

1. K. J. in 't Hout, J. Bierkens, A. P. C. van der Ploeg, J. in 't Panhuis, A semi closed-form analytic pricing formula for call options in a hybrid Heston-Hull-White model, Proc. 58th European Study Group Mathematics with Industry, eds. R.H. Bisseling et. al., Utrecht (2007).

2. K. J. in 't Hout \& S. Foulon, ADI finite difference schemes for option pricing in the Heston model with correlation, Int. J. Numer. Anal. Mod. 7 (2010) 303-320.

3. K. J. in 't Hout \& C. Mishra, Stability of the Modified Craig-Sneyd scheme for two-dimensional convection-diffusion equations with mixed derivative term, submitted for publication (2010).

4. K. J. in 't Hout \& B. D. Welfert, Unconditional stability of second-order ADI schemes applied to multi-dimensional diffusion equations with mixed derivative terms, Appl. Numer. Math. 59 (2009) 677-692.

5. J. C. Hull, Options, Futures and Other Derivatives, 6th ed., Prentice Hall, New Jersey, 2006.

6. S. E. Shreve, Stochastic Calculus for Finance II, Springer, New York, 2004.

7. D. Tavella \& C. Randall, Pricing Financial Instruments, Wiley, New York, 2000. 